\title{
Psychological evaluation in childhood migraine - steps towards a psychological profile of the child migraineur
}

\author{
Smaranda Antonia Nita ${ }^{1,2}$, Raluca Ioana Teleanu ${ }^{1,3}$, Ovidiu Alexandru Bajenaru ${ }^{1,2}$

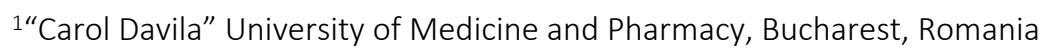 \\ ${ }^{2}$ Neurology Department, University Emergency Hospital, Bucharest, Romania \\ ${ }^{3}$ Pediatric Neurology Department, "Dr. Victor Gomoiu" Children's Hospital, Bucharest, Romania
}

\begin{abstract}
Migraine can affect children and have an impact on their quality of life. Psychological and psychiatric comorbidities have been identified over the years, in association with migraine.

Objective. To evaluate the possible relations between migraine and emotional disorders, in children.

Material and methods. Eighteen patients aged 5-17 were recruited for the migraine group and sixteen age matched patients with no criteria for migraine or any underlying organic disorder, who were diagnosed with emotional disorders, were enrolled in the control group.

The patients underwent psychological evaluation, which included use of projective diagnostic tests (tree, family and person drawings) and the ASEBA - CBCL/ YSR questionnaires, the results of which were noted in the form of raw scores, percentiles and $T$ scores, and the results were analysed for anxiety and introversion scales.

Outcomes. For the anxiety scale, the mean ASEBA score was lower in migraine patients, compared to controls $(p=0.083)$, while the percentiles on the same scale were similar between the two groups $(p=0.480)$. For the introversion scale, the mean ASEBA score was lower in the migraine group, compared to controls $(p=0.059)$ and percentiles were lower in the migraine group $(p=0.036)$.

Conclusions. Clinical observations and scores recorded through the ASEBA questionnaires pleaded for a psychological profile of migraine patients, yet the statistical analysis did not reveal significant results in this direction. However, the study opens up a road towards a more thorough assessment of migraine pediatric patients, which should include a psychological evaluation.
\end{abstract}

Keywords: migraine, child, emotional, disorder

\author{
Abbreviations \\ ASEBA - Achenbach System of Empirically Based Assessment \\ Asymptotic Sig. - Asymptotic Significance \\ CBCL - Child Behavior Checklist \\ CT - Computed Tomography \\ ENT - Ear, Nose, Throat \\ IQ - Intelligence Quotient \\ MR - Magnetic Resonance
}

\section{INTRODUCTION}

Migraine is a recurrent episodic primary headache disorder, which, depending on its type, associates symptoms like nausea, vomiting, photophobia, phonophobia, visual abnormalities, sensory dysfunction, vertigo or transient motor deficits. Mi-
MRI - Magnetic Resonance Imaging

ROC - Receiver Operator Characteristic

SEM - Standard Error of Mean

SPSS - Statistical Package for the Social Sciences

Std. Error - Standard Error

YSR - Youth Self-Report

graine is a condition which affects adults, as well as children, some of the migraine associated syndromes being diagnosed even in infancy.

The most recent classification of headache disorders, The International Classification of Headache Disorders, Third Edition, beta (1) gives a 
comprehensive view of all types of migraine and of the episodic syndromes associated with migraine, with their diagnosis criteria. Thus, migraine can be classified into the following:

- Migraine without aura

- Migraine with aura

- Migraine with typical aura

- Migraine with brainstem aura

- Hemiplegic migraine

- Familial hemiplegic migraine

- Sporadic hemiplegic migraine

- Retinal migraine

- Chronic migraine

- Episodic syndromes that may be associated with migraine

- Recurrent gastrointestinal disturbance

- Cyclical vomiting syndrome

- Abdominal migraine

- Benign paroxysmal vertigo

- Benign paroxysmal torticollis

The pathophysiology of migraine is still under debate, with numerous theories having been published over the years (2). The current understanding of migraine places it into a multifactorial category, with genetic factors being responsible for neuro-glial hyperexcitability which can lead to cortical spreading depression, which in turn, activates the trigeminovascular system, responsible for the symptoms and signs associated with migraine (3).

However, this multifaceted pathophysiologic picture is incomplete if we do not take into account the psychological factors associated with migraine. There is a debate whether these psychological factors are a contributing cause or an effect of migraine in the patients` lives. Harold G. Wolff, a pioneer in the study of migraine causes, who was the main investigator of the vascular theory, but also being interested in the psychosomatic nature of migraine and being himself a migraineur, wrote a description of migraine patients in terms of psychological typology, characterizing them as "ambitious, perfectionists, efficient and successful” (4). Since then, numerous articles have been published on the association of migraine and psychiatric disorders, mainly in adults but also in children, depression and anxiety disorders being the most common conditions associated with headache and migraine (5). Doctor Oliver Sacks, a neurologist and author of many books on the clinical picture and inside world of neurological patients, in his book "Migraine", offers a broad account of the psycho-emotional factors in migraine patients and of the psychiatric comorbidities they experience, placing an accent on psychosomatics and the organism's pattern of dealing with inner conflicts (6).

Bearing in mind the above-mentioned ideas, this article delineates the psychological traits of pediatric patients diagnosed with migraine, in search for a possible psychological profile of the child migraineur.

\section{OBJECTIVE}

This study aimed to evaluate the possible relations between migraine and emotional disorders, in children.

\section{MATERIAL AND METHODS}

A prospective study was carried out on eighteen patients recruited for the migraine group. The patients were aged five to seventeen and were diagnosed with migraine with aura or migraine without aura, according to the International Classification of Headache Disorders, third edition (beta version) (1).

The control group consisted in sixteen matched by age patients, with chronic headache syndromes, with no migraine criteria.

All patients underwent cerebral MRI with angiography and/or cerebral CT, an ENT consult and an ophthalmologic evaluation with fundoscopy, to rule out other structural, vascular or associated causes of headache. All of the patients were evaluated electroencephalographically to observe eventual epileptiform abnormalities, in the context of migraine or related to an epileptic nature of the recurrent headache episodes and/or of the visual or sensory accompanying phenomena.

The patients and their parents had an interview with the clinical psychologist, who evaluated the childrens' behavior independently and in relation to the relevant persons in their lives, their adequacy in expressing their emotions and inner needs, their emotional reactivity to different experiences, the existence of emotional blockages. The tools used were projective diagnostic tests (tree, family and person drawings) and the ASEBA - CBCL/ YSR 
(Achenbach System of Empirically Based Assessment Child Behavior Checklist/Youth Self-Report) questionnaires, the results of which were noted in the form of raw scores, percentiles and $\mathrm{T}$ scores, and the results were analysed mainly for anxiety and introversion scales. Percentiles above 93 were considered clinically significant, The patients were also evaluated cognitively using the Raven Standard Progressive Matrices and their level of attentiveness and concentration on tasks were observed.

The results were collected via Microsoft Excel 2010 and statistically analyzed using SPSS software 21.0.

Quantitative numerical variables were expressed as mean $+/$ - standard error of mean (SEM). They were analyzed using appropriate tests: T-Test or One-Way Anova Test, as well as Mann-Whitney (U-Test) and Two-Sample Kolmogorov-Smirnov Tests, for small groups of patients.

The distribution was analyzed with Pearson Chi - square Test. The Mantel-Haenszel Common Odds Ratio Test evaluated the odd ratio.

Parametric corellation was made, using the Pearson correlation coefficient and the associated p-value. The Pearson correlation coeffcient was interpreted according to the Salkin scale: values between $\pm 0,8$ - 1 - very strong, values between $\pm 0,6$ 0,8 - stong, between 0,4-0,6 - moderate, between 0,2-0,4 - low, between 0- 0,2 - no correlation. A p-value of $<0.05$ was considered significant.

\section{OUTCOMES}

Migraine was were more frequent in male patients $(68.8 \%)$, compared to female patients $(38.9 \%) ; p=0.082$.

The mean age of the patients was similar between the two groups (migraine/control; $p=0.788$ ): 12 and 12.25 years, respectively.

The age of the patients from the migraine group correlates $(p=0.023)$ with migraine type and patient gender.

Clinical observations during the psychological evaluations revealed, especially in the migraine group, common traits, pointing towards a psychological profile of the child diagnosed with migraine, consisting in: marked tendency towards anxiety, with negative anticipation, over - analysis, rumination and a considerable fear of failure, predomi- nately in report to newly encountered situations. While having a low self-esteem, children were found to interiorize and repress their emotions and personal needs, while struggling to meet other people's expectations and thus to avoid a possible rejection. This went on to the extent of displaying a marked auto - critical and self - blaming behavior, if they felt they had not met these expectations. They had a pronounced duty sense in relation to their parents, a marked tendency towards perfectionism and cognitive performance anxiety.

For the anxiety scale, the mean ASEBA score was lower in migraine patients, compared to controls $(\mathrm{p}=0.083)$, while the percentiles on the same scale were similar between the two groups $(\mathrm{p}=$ $0.480)$. The mean ASEBA score $(p=0.111)$, percentiles $(p=0.245)$ and $T$ score $(p=0.230)$ were higher in patients diagnosed with migraine with aura, compared to those with migraine with aura. The $\mathrm{T}$ score for anxiety was similar between the migraine and control groups $(\mathrm{p}=0.471)$.

For the introversion scale, the mean ASEBA score was lower in the migraine group, compared to controls $(p=0.059)$ and percentiles were lower in the migraine group $(\mathrm{p}=0.036)$. However, The ASEBA score, as well as the percentiles and $T$ scores $(p=0.148)$, on the same scale, were higher in migraine with aura patients. The $\mathrm{T}$ score for introversion was lower in patients with migraine, compared to the control group $(\mathrm{p}=0.080)$.

All patients had a normal intellect. The mean IQ (Intelligence Coefficient) was similar between the migraine and control groups $(p=0.926)$; however, it was lower in patients with migraine with aura, compared to those with migraine without aura $(\mathrm{p}=$ $0.166)$.

Age had a moderate, statistically significant negative correlation with the IQ $(\mathrm{r}=0.434, \mathrm{p}=$ 0.015), a positive correlation with the ASEBA anxiety score $(r=0.413, p=0.021)$ and a positive correlation with the ASEBA Introversion score $(\mathrm{r}=$ $0.327, \mathrm{p}=0.072$ ).

\section{ROC (Receiver Operator Characteristic) curves for emotional disorder associated parameters}

Analysis of the ROC curves shows that the negative variation of the ASEBA anxiety score defines 


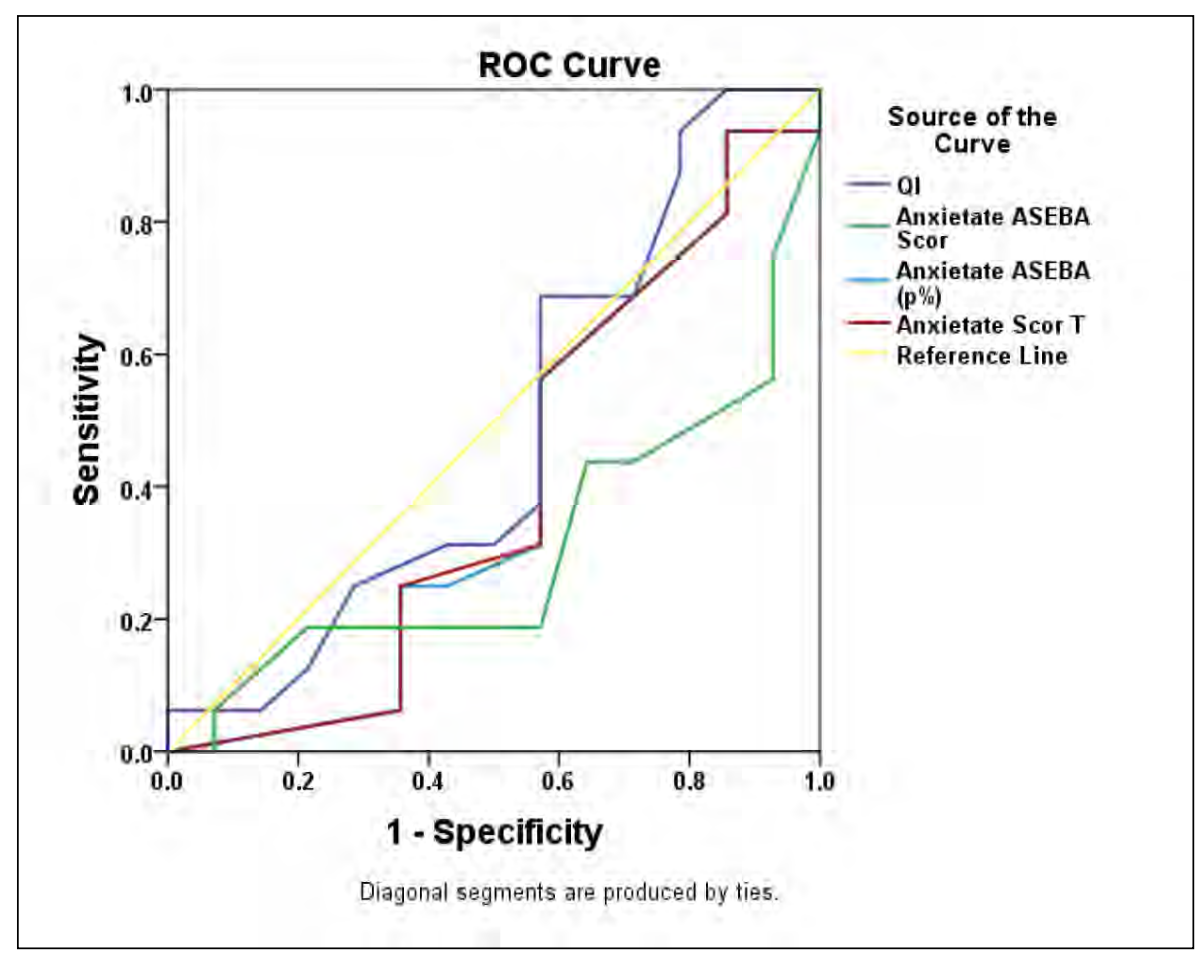

FIGURE 1. ROC curves for emotional parameters, ASEBA anxiety scale

TABLE 1. Area under the ROC curve for emotional parameters, ASEBA anxiety scale

\begin{tabular}{|c|c|c|c|c|c|}
\hline \multicolumn{6}{|c|}{ Area under the curve } \\
\hline \multirow[t]{2}{*}{ Test Result Variable(s) } & \multirow[t]{2}{*}{ Area } & \multirow{2}{*}{$\begin{array}{l}\text { Std. } \\
\text { Error }\end{array}$} & \multirow{2}{*}{$\begin{array}{l}\text { Asymptotic } \\
\text { Sig. }\end{array}$} & \multicolumn{2}{|c|}{$\begin{array}{l}\text { Asymptotic } 95 \% \\
\text { Confidence Interval }\end{array}$} \\
\hline & & & & Lower Bound & Upper Bound \\
\hline IQ & 0.482 & 0.111 & 0.868 & 0.264 & 0.700 \\
\hline Anxiety ASEBA Score & 0.306 & 0.099 & 0.071 & 0.112 & 0.499 \\
\hline Anxiety ASEBA (p\%) & 0.400 & 0.108 & 0.350 & 0.187 & 0.612 \\
\hline Anxiety T Score & 0.402 & 0.108 & 0.360 & 0.189 & 0.614 \\
\hline
\end{tabular}

the migraine diagnosis, with a borderline statistic significance (green curve, aria $=0.306,95 \% \mathrm{CI}$ 0.112-0.499, $\mathrm{p}=0.071)$. Low values of the ASEBA anxiety score are associated with migraine (Figure 1; Table 1).

An anxiety diagnosis, defined by an ASEBA anxiety percentile above 93 , was questioned. The Pearson Chi - square Test showed there was no significant ( $p=0.739)$ correlation between anxiety (as defined above) and migraine, when compared to the control group, anxiety being present in $80 \%$ of patients with migraine and in $75 \%$ of controls. The Mantel - Haenszel Common Odds Ratio Estimate showed that there was a minor risk (odd ratio), of 0.750 , of having anxiety if the patient was diagnosed with migraine, compared to the control group $(\mathrm{OR}=0.750 \pm 0.866,95 \%$ CI $0.137-4.095, \mathrm{p}=$ $0.740)$.
Negative variation of the introversion ASEBA score defines the migraine diagnosis, with a borderline statistic significance (red curve, aria $=0.300$, $95 \%$ CI 0.112-0.488, $\mathrm{p}=0.058$ ). Low values of the ASEBA introversion score are associated with migraine (Figure 2; Table 2).

\section{CONCLUSIONS}

Migraine is a rare disorder in children, due to the necessity of a thorough and complete differential diagnosis.

Although there are many research directions in migraine pathophysiology, placing it in a multifactorial category of disorders, and although psychological factors have been known to be associated with headache syndromes, there is increasing evidence that persons suffering from migraine may 


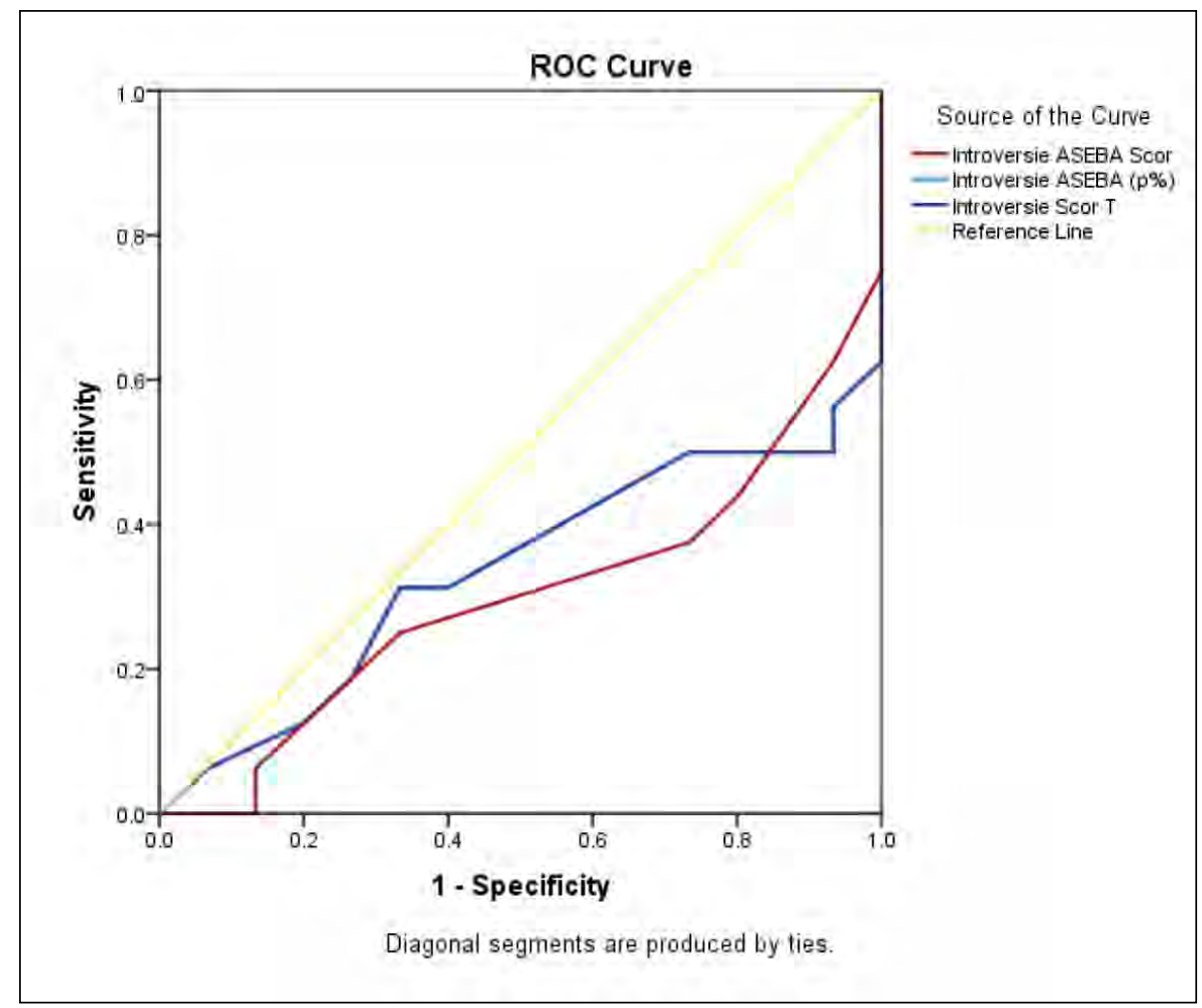

FIGURE 2. ROC curves for emotional parameters, ASEBA introversion scale

TABLE 2. Area under the ROC curve for emotional parameters, ASEBA introversion scale

\begin{tabular}{|l|c|c|c|c|c|}
\hline & & & Area under the curve \\
\hline & Area & Std. Error & $\begin{array}{c}\text { Asymptotic } \\
\text { Sig. }\end{array}$ & \multicolumn{2}{c|}{$\begin{array}{c}\text { Asymptotic 95\% Confidence } \\
\text { Interval }\end{array}$} \\
\cline { 5 - 6 } & & & $\mathbf{0 . 0 5 8}$ & 0.112 & 0.488 \\
\hline Introversion ASEBA Score & 0.300 & 0.096 & 0.123 & 0.140 & 0.535 \\
\hline Introversion ASEBA (p\%) & 0.338 & 0.101 & 0.123 & 0.140 & 0.535 \\
\hline Introversion T Score & 0.338 & 0.101 & 0.123 \\
\hline
\end{tabular}

have distinct psychological traits of personality and behavior. While stress is a common entity underlying many pain syndromes, the idea of particular models of reaction to stress, in migraine, is emerging.

This study aimed to analyse the psychological traits of children diagnosed with migraine. Although clinical observations and the scores recorded through the ASEBA questionnaires pleaded for an anxiety predominant psychological profile of migraine patients, statistical analysis did not reveal significant results in this direction, when data was compared to values from controls. It should be mentioned that the control group consisted in similarly aged patients evaluated in the same center, for recurrent headache, which could not be attributed to migraine nor organic disorders and who were discharged with a diagnosis of emotional disorder.
Thus, the existence of emotional factors associated with headache in the control group, could have resulted in a weak comparison model for migraine patients. Moreover, the small number of enrolled patients could also account for the paucity of statistically significant results.

The present study opens up a road towards a more thorough assessment of migraine pediatric patients, which should include psychological evaluation, with objective measurements on relevant scales. A broader study, on large cohorts of patients of pediatric age diagnosed with migraine, which should also take into consideration the results of psychological counseling and psychotherapy, could shed more light into the yet incomplete pathophysiological picture of migraine, on behavioural development and on the evolution of symptoms from young age into adulthood. 


\section{Acknowledgements}

Acknowledgements to the Pediatric Neurology Department, "Dr. Victor Gomoiu" Hospital,
Bucharest, where the patients were admitted and the clinical examinations and paraclinical tests took place.

Conflict of interest: none declared Financial support: none declared

\section{REFERENCES}

1. Headache Classification Committee of the International Headache Society, The International Classification of Headache Disorders, 3rd edition (beta version). Cephalalgia, 2013;33 (9):629-808.

2. Goadsby PJ. Pathophysiology of migraine. Ann Indian Acad Neurol. 2012; 15(Suppl 1):S15-22.

3. Swaiman K, Ferriero D, Ashwal S et al. Swaiman's Pediatric Neurology, Principles and Practice, Fifth Edition, Elsevier, 2012; 1:883-886.

4. Akkermans R. Harold G Wolff. The Lancet Neurology, 2015; 14(10):982-983.

5. Sheftell F.D, Atlas SJ. Migraine and psychiatric comorbidity: From theory and hypotheses to clinical application. Headache, 2002; 42(9):934-4.

6. Sacks O. Migraine. 2012, Picador, 211-228. 